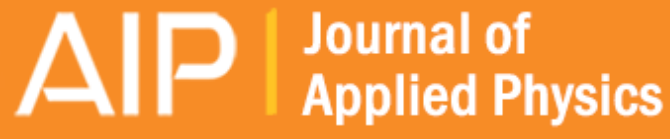

\section{The Impact of a Body on a Water Surface at an Arbitrary Angle}

Leon Trilling

Citation: Journal of Applied Physics 21, 161 (1950); doi: 10.1063/1.1699617

View online: http://dx.doi.org/10.1063/1.1699617

View Table of Contents: http://scitation.aip.org/content/aip/journal/jap/21/2?ver=pdfcov

Published by the AIP Publishing

\section{Articles you may be interested in}

Impact force measurement of a spherical body dropping onto a water surface

Rev. Sci. Instrum. 85, 075108 (2014); 10.1063/1.4878629

Water Drop Impact on Cold Surfaces with Solidification

AIP Conf. Proc. 1376, 451 (2011); 10.1063/1.3651944

Use of empirical many-body potentials in the simulation of cluster impacts on surfaces

AIP Conf. Proc. 392, 487 (1997); 10.1063/1.52507

Extension of the surface variational principle to arbitrary motion of bodies of revolution.

J. Acoust. Soc. Am. 91, 2417 (1992); 10.1121/1.403206

Compressibility Effects in the Problem of Body Impact on a Free Surface

J. Acoust. Soc. Am. 37, 1190 (1965); 10.1121/1.1939467

\section{A|P $\left.\right|_{\text {Applied Physics }} ^{\text {Journal of }}$}

Journal of Applied Physics is pleased to announce André Anders as its new Editor-in-Chief 
Mach angle. It is also not inconsistent if the local mean angle of attack of the airfoil is zero at all points. Supersonic edges throughout are not a necessary condition for the existence of a solution. There is some indication that nonexistence of a solution may require that portions of the leading edge be downstream and in the Mach direction relative to portions of the trailing edge. It seems that it has not yet been determined rigorously or finally under what conditions the two edges may be prescribed to serve as separate line and rejoining line, respectively, without excluding a solution of Eq. (1), and under what conditions they may not.

\section{BIBLIOGRAPHY}

L. Prandtl, "Theorie des Flugzeugtragflügels im zusammendrückbaren Medium," Luftfahrt-Forschung 13, 313-319 (1936).

P. A. Lagerstrom, "Formulas in Three Dimensional Wing Theory," Douglas Aircraft Company, Report No. SM 11901 (July 8, 1946).

Th. von Karman, "Supersonic Aerodynamics," J. Aero. Sci. 14, 373-402 (1947). See Section 5d.

\title{
The Impact of a Body on a Water Surface at an Arbitrary Angle
}

\author{
LeON TriLling* \\ Hydrodynamics Laboratory, California Insitute of Technology, Pasadena, California
}

(Received August 18, 1949)

\begin{abstract}
This paper presents an approximate method of determining the pressure distribution during impact on the surface of a body which strikes a horizontal water surface at an arbitrary angle.

The effect of the splash is neglected and the pressure on the free boundary is assumed proportional to the potential, as if the process were an impulse. The shape of the submerged portion of the striking body is approximated by a semi-ellipse (two dimensions), by a hemisphere, half an ellipsoid of revolution and half a general ellipsoid. Under those conditions, explicit results for the pressure distribution are found. As a special example, the impact pressures on a sphere striking at $45^{\circ}$ angle are computed in detail.
\end{abstract}

A $\mathrm{N}$ estimate of the pressure forces on a body which strikes a water surface at an arbitrary angle is important in many problems, such as the design of seaplane hulls or naval ballistics.

The present work discusses the earliest stage of the water entry phenomenon which precedes the appearance of a cavity and displays the highest pressures. It is usually called the impact stage.

The problem of water impact has been discussed by numerous writers interested in the landing characteristics of seaplanes and the underwater trajectory of projectiles. Because of the difficulty of the problem, they were forced to various simplifying assumptions. Thus, von Karman ${ }^{1}$ approximated the shape of the striking body by a growing flat plate in the plane of the water surface. $\mathrm{H}$. Wagner ${ }^{2}, 3$ refined Karman's analysis by making some allowance for the water splash, and M. S. Plesset ${ }^{4}$ by replacing the flat plate by an elliptic disk. To take into account the penetration of the body into the water, Schiffman and Spencer ${ }^{5,6}$ approximated

\footnotetext{
* The major portion of this study was carried out while the writer was with the Naval Ordnance Test Station; the writer also wishes to acknowledge the support of the ONR.

${ }^{1}$ Th. von Karman, "The impact on sea-plane floats during landing," NACA-TN 321 (1929).

${ }^{2} \mathrm{H}$. Wagner, "On the landing of seaplanes," ZFM (January, 1931)-NACA-TM 622

${ }^{3} \mathrm{H}$. Wagner, "Uber stoss und gleitvorgange an der oberflache von flussigkeiten," ZAMM, 12, 193-215 (1932).

${ }^{4} \mathrm{M}$. S. Plesset, "An investigation of the impact forces on torpedoes entering water," Douglas Company Report SM3937 (December, 1942).

"Schiffman and Spencer, "The force of impact on a sphere striking a water surface," AMP-421R-AMG-NYU No. 105 (February, 1945).

Schiffman and Spencer, "The force of impact on a sphere
}

its shape by a spherical lens; P. Y. Chou ${ }^{7}$ approximated it by a spherical bowl, whose potential was calculated by A. B. Basset. ${ }^{8}$ Those latter investigations give accurate results in the case of normal impact, but they are too complicated to be generalized to impact at an arbitrary angle. Some simple two-dimensional problems were solved in all generality by L. I. Sedoff ${ }^{9}$ who used the powerful methods of conformal transformation.

In the following pages, the problem is linearized and the classical methods of potential theory are applied to determine the pressure on two-dimensional bodies whose submerged portion may be approximated by a semi-ellipse, and on three-dimensional bodies which may be approximated by a hemisphere, half an ellipsoid of revolution, and half a general ellipsoid. Because of the linear nature of the simplified problem the effect of the vertical and horizontal components of impact velocity may be discussed separately and superimposed, so that one obtains results valid for any angle of impact.

\section{STATEMENT OF THE PROBLEM}

A three-dimensional half-space filled with incompressible fluid of density $\rho$ at rest with a horizontal free

striking a water surface (2)," AMP-42.2R-AMG-NYU No. 133 (July, 1945)

${ }^{7}$ P. Y. Chou, "On impact of spheres upon water," U.S. NOTS (1946), (to be published)

${ }^{8} \mathrm{~A}$. B. Basset, "On the potential of an electrified spherical bowl and on the velocity potential due to the motion of an infinite liquid about such a bowl," Proc. Math. Soc. London, 1st Series 16, 286-306 (1885).

9 L. I. Sedoff, "The impact of a solid body which moves at the surface of an incompressible fluid," TCAHI Report No. 187, Moscow (1934). 
surface, is struck by a body $S$ which travels at a velocity $\bar{V}$, at an angle $\alpha$ from the horizontal plane. The fluid in the vicinity of the impact is set into motion; a small amount rises above the horizontal plane and forms a splash; a larger quantity is disturbed below the free surface, which is no longer horizontal, to make room for the body. The force of gravity, the hydrostatic force, viscous drag forces, surface tension forces, and the hydrodynamic forces all act on the body. But if the velocity $\bar{V}$ is large, during the impact stage, the hydrodynamic forces alone are significant, and the other forces are neglected in all that follows.

The hydrodynamic force is equal to the rate of change of momentum of the fluid set into motion by the impact. Since the fluid is assumed to be non-viscous and the motion is started from rest, the flow is irrototional and the velocity field may be derived from a potential $\Phi$. The force is therefore:

$$
\bar{F}=\frac{\rho}{\bar{V}} \frac{d}{d t} \int_{S+S^{\prime}+S^{\prime \prime}} \Phi \frac{\partial \Phi}{\partial n} d S .
$$

The integration is carried out over the boundaries of the fluid.** The forces acting on the body are therefore computed by a quadrature if the flow potential is known. Since the impact occurs in a very short time interval, $\bar{V}$ is assumed constant.

The velocity potential $\Phi$ of an incompressible perfect fluid satisfies the Laplace equation:

$$
\Delta \Phi=0
$$

and certain conditions along the boundary of the fluid. The disturbance due to the impact must disappear far from the point of impact. Thus, if $r$ denotes the distance from the point of impact, one has

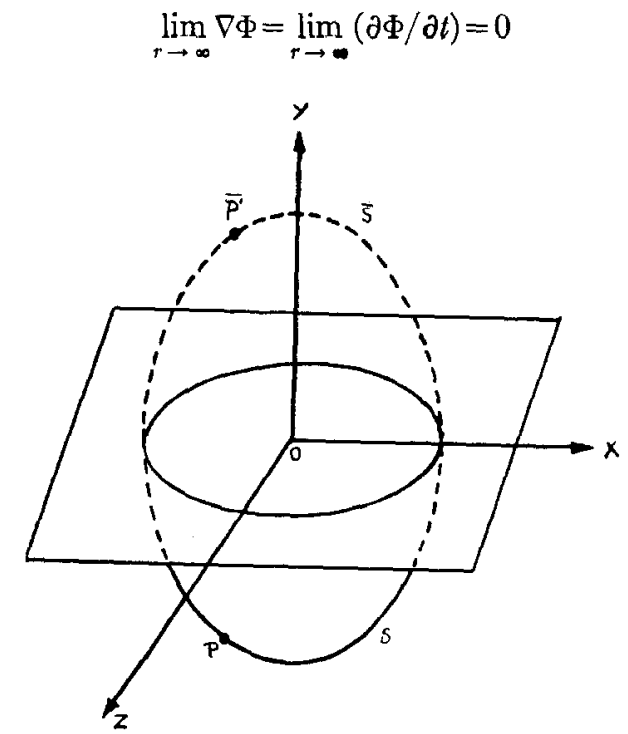

FiG. 1. General boundary conditions.

** $S$ represents the surface of the submerged portion of the body, $S^{\prime}$ the free water surface, and $S^{\prime \prime}$ is a hemisphere of infinitely large radius with center at the body. or the equivalent condition

$$
\lim _{r \rightarrow \infty} \Phi=0 .
$$

The velocity component normal to the surface of the impinging body must vanish:

$$
\left.\frac{\partial \Phi}{\partial n}\right)_{S}=-V \cos \lambda,
$$

where $n$ is the direction normal to the wetted surface $S$, and $\lambda$ is the angle between that normal and the direction of $\bar{V}$. Finally, along the free surface, the pressure in the fluid must equal atmospheric pressure, which may be taken equal to zero. This gives a condition on $\Phi$, when Euler's equation is used:

$$
(\partial \bar{u} / \partial t)+\bar{u} \cdot \nabla \bar{u}=(-1 / \rho) \nabla p,
$$

where $\tilde{u}$ is the velocity vector in the fluid and $p$ the pressure. An integration brings this equation into the form

$$
-\partial \Phi / \partial t+\frac{1}{2}(\nabla \Phi)^{2}=-(p / \rho)+F(t) .
$$

Boundary condition (1.3) applied on the free surface where $p$ vanishes, gives $F(t) \equiv 0$, so that the boundary condition valid on the free surface is:

$$
\frac{1}{2}(\nabla \Phi)^{2}-(\partial \Phi / \partial t)=0 .
$$

One is thus led to investigate solutions of (1.2) subject to boundary conditions (1.3a, 1.4, 1.5b). Since (1.5b) is not linear, and the surface where it holds must be determined from the solution, this problem is extremely difficult; indeed, it has not been solved.

In linearizing boundary condition (1.5b), one argues that the impact occurs during a very short time interval, and may therefore be considered as an impulse. But it is known ${ }^{10}$ that in the case of impulsive motion, the dynamic pressure is proportional to the velocity potential. Since the pressure vanishes on the free surface, one replaces condition (1.5b) by the more restrictive condition

$$
\Phi\left(S^{\prime}\right)=0,
$$

where $S^{\prime}$ denotes the free surface. It is further assumed that the effect of the deformation of the free surface due to the splash is small, so that (1.5c) is applied along the original free surface:

$$
\Phi(x, 0, z, t)=0
$$

in a cartesian system with origin at the center of impact, where the $y$ axis is normal to the undisturbed free surface and the vector $\bar{V}$ lies in the plane $z=0$.

The problem of finding the potential $\Phi$ which satisfies Eq. (1.2) with boundary conditions (1.3a, 1.4, 1.5d) is a linear harmonic problem. It is convenient to investigate the potentials due to the various components

${ }^{10} \mathrm{H}$. Lamb, Hydrodynamics (Dover Publications, New York, 1944), sixth edition, London, (1932). 
of $\bar{V}$ separately and to superimpose the results. Thus:

$$
\Phi=V_{x} \varphi_{1}+V_{y} \varphi_{2}+V_{z} \varphi_{3}+\Omega_{y z} \varphi_{4}+\Omega_{z x} \varphi_{5}+\Omega_{x y} \varphi_{6}
$$

where $V_{x, y, z}$ represent the $x, y, z$ components of the velocity vector $\bar{V}$ and $\Omega_{i j}$ represent rotation velocities about the axes. In the problems to follow, $V_{z}, \Omega_{i j}$ vanish identically, so that

$$
\Phi=V\left(\varphi_{1} \cos \alpha+\varphi_{2} \sin \alpha\right) .
$$

If the symmetric image $\bar{S}$ of the wetted surface $S$ with respect to the plane $y=0$ is constructed, (Fig. 1) and the field of flow is continued into the upper halfspace, it follows from condition (1.5d) and the harmonic character of $\Phi$ that :

$$
\Phi(x, y, z, t)=-\Phi(x,-y, z, t) .
$$

The velocity components $u, v, w$, parallel to the $x, y, z$, axes, therefore have the following symmetry properties:

$$
\begin{aligned}
u(x, y, z) & =-u(x,-y, z) \\
v(x, y, z) & =v(x,-y, z) \\
w(x, y, z) & =-w(x,-y, z)
\end{aligned}
$$

or, if the velocities normal to the surface $S+\bar{S}$ at two symmetric points $P, \bar{P}$ are compared,

$$
(\partial \varphi / \partial n)_{P}=-(\partial \varphi / \partial n)_{\bar{P}}
$$

If $\lambda_{1}, \lambda_{2}$ denote the angle between the normal to the surface $S+\bar{S}$ and the $x, y$ axes, it follows from (1.7d) that boundary conditions $(1.5,1.4)$ become, for the potentials $\varphi_{1}, \varphi_{2}$

$$
\begin{aligned}
& -\partial \varphi_{1} / \partial n=\cos \lambda_{1}(|y| / y) \\
& -\partial \varphi_{2} / \partial n=\cos \lambda_{2}
\end{aligned}
$$

these conditions being valid in the entire space.

The problem is thus split into two parts. The velocity component normal to the horizontal free surface induces the same potential as that of a fully submerged body in translation. The velocity component parallel to the horizontal free surface induces the potential which would obtain if the fluid above the plane of symmetry were flowing with a velocity equal and opposite that of the fluid below, causing a vortex sheet along the plane of symmetry.

One is now ready to express the momentum integral in terms of $\varphi_{1}$ and $\varphi_{2}$ :

$$
\begin{aligned}
& \frac{\rho}{V} \int_{S} \Phi \frac{\partial \Phi}{\partial n} d S^{\prime}=\rho V\left[\mathrm{i} \cos ^{2} \alpha \int_{S} \varphi_{1} \frac{\partial \varphi_{1}}{\partial n} d S\right. \\
& \quad+\sin \alpha \cos \alpha\left(\mathrm{i} \int_{S} \frac{\partial \varphi_{1}}{\varphi_{2}} d S+\mathbf{j} \int_{S} \varphi_{1} \frac{\partial \varphi_{2}}{\partial n} d S\right) \\
& \left.\quad+\mathbf{j} \sin ^{2} \alpha \int_{S} \frac{\partial \varphi_{2}}{\partial n} d S\right]=\mathrm{i} M_{x}+(\mathbf{i}+\mathbf{j}) M_{x y}+\mathbf{j} M_{\nu}
\end{aligned}
$$

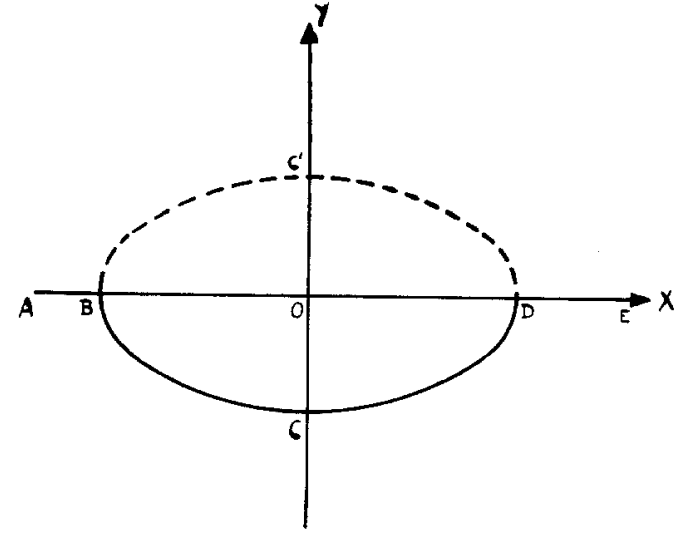

FIG. 2. Water impact of an elliptic cylinder.

where $\mathbf{i}$ and $\mathbf{j}$ are unit vectors parallel to the $x, y$ axes. Note that $\varphi_{2}$ is positive over the entire surface $S$. On the other hand, $\varphi_{1}$ may be negative over part of the rear-half of the surface. If $S$ is symmetric with respect to the plane $x=0, \varphi_{1}$ is antisymmetric and negative over the entire rear-half. But the dynamic pressure $p=\rho \Phi$ cannot become negative, since this would imply that the fluid is in tension and therefore separates from the body surface to form a cavity. Actually, because of the action of viscous forces and surface tension forces, separation is not instantaneous, and during the short period of time in which the impact occurs, the streamline pattern is not changed appreciably. But the integration in (1.9) must be carried out only over regions where $\varphi=\varphi_{1} \cos \alpha+\varphi_{2} \sin \alpha$ is positive.

The simplified problem formulated above does not depend on the time parameter directly since the equation of motion (1.2) and its boundary condition (1.3, 1.8) do not involve time variations. But as time passes, an increasing volume of the striking body is immersed in the fluid. Passage of time is, therefore, marked by a change in the dimensions and shape of the immersed body. Translation of the body in a horizontal direction does not change the shape of the immersed body and induces no force, but translation perpendicular to the free surface does.

In order to compute the time rate of change of momentum, therefore, it is necessary to determine how the immersed portion of the body changes with time. Most bodies whose impact may be of interest (projectiles, seaplane floats, etc.) have immersed portions of shapes so complicated that an analytic determination of $\varphi_{1}, \varphi_{2}$ is not possible. Following the scheme of previous calculations, one is led to approximate the immersed portion of the striking body at any time by some geometrically simple body (hemisphere, halfellipsoid, etc.). The simple shape is defined by a certain number of parameters: the sphere by its radius, the ellipsoid of revolution by two axes, the general ellipsoid by three axes, etc. The approximating process consists in selecting certain properties of the actual body and defining the parameters of the approximating body so 
that those properties are preserved. One can generally preserve as many properties as there are independent parameters defining the approximating body. For instance, if at any time $t$, the immersed portion of the striking body is approximated by a half-ellipsoid, the three axes of the ellipsoid are chosen so that the depth of penetration, the length and the immersed volume of the half-ellipsoid equal those of the actual body. As time passes, the depth of penetration, length and immersed volume of the actual body change, and the three axes of the approximating half-ellipsoid change correspond-

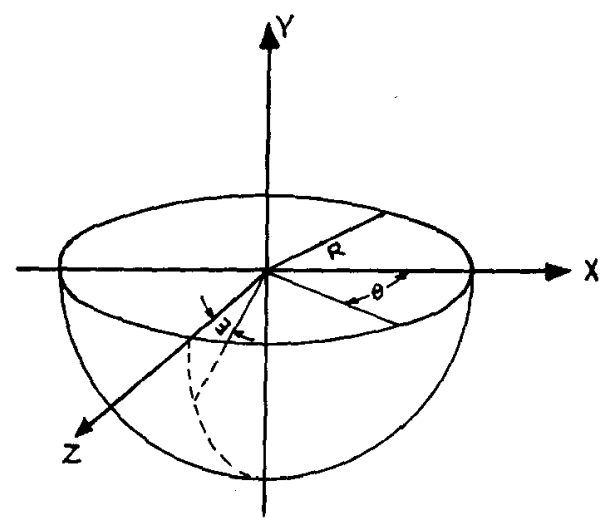

FIG. 3. Water impact of a sphere.

ingly. The three axes of the ellipsoid are, therefore, known functions of time, when the motion of the actual body is known, either from measurements or from calculations. The momentum integral $M(1.9 \mathrm{a})$ of the approximating body depends only on its geometrical parameters $\alpha_{1}, \alpha_{2}, \alpha_{3} \cdots \alpha_{i}$, which are known functions of time, since $V$ and $\rho$ are constant. The drag force is therefore given by:

$$
\begin{aligned}
F_{x}=\rho V \cos \alpha \sum\left(d \alpha_{i} / d t\right) \\
\times\left[\left(\partial M_{x} / \partial \alpha_{i}\right) \cos \alpha+\left(\partial M_{x y} / \partial \alpha_{i}\right) \sin \alpha\right] \\
F_{y}=\rho V \sin \alpha \sum\left(d \alpha_{i} / d t\right) \\
\times\left[\left(\partial M_{x y} / \partial \alpha_{i}\right) \cos \alpha+\left(\partial M_{y} / \partial \alpha_{i}\right) \sin \alpha\right] .
\end{aligned}
$$

The problem of finding approximately the forces acting on a given body when it strikes a horizontal surface is, therefore, investigated as follows:

An approximating body is selected and its parameters $\alpha_{i}$ are computed as functions of time from the known behavior of the actual body.

The potential problem for the approximating body is solved and the momentum integral is computed in terms of $\alpha_{i}$.

The force function is found by application of Eqs. (1.10.)

The second step of this procedure is the most important and difficult. It can be carried out once and for all for several approximating body shapes. Then the results can be applied to a wide variety of problems by the proper fitting procedure.

In the following, the potential problem is solved for an infinitely wide semi-elliptic cylinder (two-dimensional problem solved by Sedoff's methods) and for a sphere, an ellipsoid of revolution and a general ellipsoid (solved by harmonic analysis methods). As an example of the fitting process, the water entry forces on a sphere striking at $45^{\circ}$ are computed by using a general ellipsoid as the approximating body.

\section{INFINITE SEMI-ELIIPTIC CYLINDER}

The first potential problem discussed in detail concerns an infinitely wide semi-elliptic cylinder of major axis $2 a$ and minor axis $2 b$, the free surface $y=0$ coinciding with the major axis (Fig. 2). The boundary conditions to be satisfied by the potentials $\varphi_{1}, \varphi_{2}$ in the entire $x, y$ plane are obtained from (1.8). If a stream function $\psi$ is introduced, then, on the body surface,

$\partial \varphi / \partial n=\partial \psi / \partial s$

$$
=\cos \alpha(\partial y / \partial s)(|y| / y)-\sin \alpha(\partial x / \partial s),
$$

where $d s$ is a length element along the surface of the ellipse. This is integrated to give:

$$
\psi=|y| \cos \alpha-x \sin \alpha .
$$

One is now required to find an analytic complex potential function $F(z)=F(x+i y)$ whose imaginary part satisfies (2.1a) on the boundary of the ellipse.

The conformal transformation

$$
-2 z=(a-b) \zeta+(a+b) 1 / \zeta
$$

transforms the ellipse in the $z$ plane into the unit circle in the $\zeta$-plane. Therefore, in the $\zeta$-plane the complex potential $F(\zeta)$ must satisfy the conditions below on the unit circle $\zeta=e^{i \theta}$ :

$$
\begin{aligned}
\psi & =b|\sin \theta| \cos \alpha+a \cos \theta \sin \alpha \\
& =\frac{2 b \cos \alpha}{\pi}\left[1-2 \sum_{1}^{\infty} \frac{\cos 2 n \theta}{4 n^{2}-1}\right]+a \sin \alpha \cos \theta .
\end{aligned}
$$

It follows that:

$$
F(\zeta)=\frac{2 b i \cos \alpha}{\pi}\left[1+2 \sum_{1}^{\infty} \frac{\zeta^{2 n}}{4 n^{2}-1}\right]+a i \sin \alpha \zeta .
$$

To sum the power series inside and on the unit circle, note that

$$
2 \sum_{1}^{\infty} \frac{\zeta^{2 n}}{4 n^{2}-1}=\zeta \sum_{1}^{\infty} \frac{\zeta^{2 n-1}}{2 n-1}-\frac{1}{\zeta} \sum_{1}^{\infty} \frac{\zeta^{2 n+1}}{2 n+1}
$$

and since

$$
\sum_{1}^{\infty} \zeta^{2 n}=\zeta^{2} / 1-\zeta^{2}
$$

one has

$$
\begin{aligned}
& \sum_{1}^{\infty} \frac{\zeta^{2 n-1}}{2 n-1}=\int_{0}^{\zeta} \frac{d \zeta}{1-\zeta^{2}}=\frac{1}{2} \log \frac{1+\zeta}{1-\zeta} \\
& \sum_{1}^{\infty} \frac{\zeta^{2 n+1}}{2 n+1}=\int_{0}^{\zeta} \frac{\zeta^{2} d \zeta}{1-\zeta^{2}}=-\zeta+\frac{1}{2} \log \frac{1+\zeta}{1-\zeta}
\end{aligned}
$$


so that

$$
F(\zeta)=\frac{i b \cos \alpha}{\pi}\left(\frac{1}{\zeta}-\zeta\right) \log \frac{1+\zeta}{1-\zeta}+i a \zeta \sin \alpha .
$$

All the manipulations carried out above are valid in the annulus $0<|\zeta|<1$ because all series and integrals converge uniformly there. It is easily verified that $F(\zeta)$ is an analytic function regular everywhere in and on the unit circle. Separating real and imaginary parts, on the circle, one finds

$$
\psi=b \cos \alpha|\sin \theta|+a \cos \theta \sin \alpha
$$

$\varphi=-\sin \theta[(2 b / \pi) \cos \alpha \log |\tan (\theta / 2)|+a \sin \alpha]$.

The boundary condition (2.3) is therefore satisfied and the pressure distribution is given by $(2.7 \mathrm{~b})$. The separation point occurs at $\theta_{s}$ defined by

or

$$
(2 b / \pi) \cos \alpha \log \left|\tan \left(\theta_{\mathrm{s}} / 2\right)\right|+a \sin \alpha=0
$$

$$
\theta_{s}=2 \tan ^{-1} \exp [-(a \pi / 2 b) \tan \alpha] \text {. }
$$

In most cases $a \gg b$ and $\tan \alpha=0(1)$; then $\theta_{s}$ is small: $\theta_{\mathrm{s}}=0\left(10^{-3}\right)$ and the separation phenomenon may be neglected. Under those circumstances, the momentum integrals are:

$$
\begin{aligned}
M_{x} & =2 b^{2} / \pi \\
M_{x y} & =M_{y x}=0 \\
M_{y} & =\pi a^{2} / 2 .
\end{aligned}
$$

\section{THE SPHERE}

Consider a sphere of radius $R$ half-submerged in a fluid (Fig. 3). The potential $\varphi=\varphi_{1} \cos \alpha+\varphi_{2} \sin \alpha$ must satisfy the equation

$$
\Delta \varphi_{1}=\Delta \varphi_{2}=0
$$

with the boundary conditions:

$$
\begin{gathered}
\lim _{r \rightarrow \infty} \varphi_{1}=\lim _{r \rightarrow \infty} \varphi_{2}=0 \\
\left(\partial \varphi_{2} / \partial r\right)(R, \theta, \omega)=-\cos \omega \\
\left(\partial \varphi_{1} / \partial r\right)(R, \theta, \omega)=-(|\cos \omega| / \cos \omega) \cos \theta .
\end{gathered}
$$

The potential $\varphi_{2}$ is immediately found:

$$
\varphi_{2}=\left(R^{3} / 2 r^{2}\right) \cos \omega .
$$

The potential $\varphi_{1}$ is determined by fitting the general harmonic function convergent for large $r$

$$
\varphi_{2}=\sum_{0}^{\infty} \sum_{0}^{n+1} \frac{1}{r^{n+1}} P_{n}^{s}(\cos \omega)\left[a_{n}^{s} \cos s \theta+b_{n}^{s} \sin s \theta\right]
$$

to satisfy boundary condition (3.3b). It is clear by inspection that $b_{n}^{s}=0$ and $a_{n}^{s>1}=0$, while the non- vanishing coefficients $a_{n}{ }^{1}$ must satisfy the relation:

$$
\begin{array}{rlrl}
\sum_{0}^{\infty} \frac{n+1}{R^{n+2}} a_{n}{ }^{1} P_{n}{ }^{1}(\cos \omega) & =\sum_{0}^{\infty} \alpha_{n} P_{n}^{1} \\
& =-1 & {[0<|\omega|<(\pi / 2)]} \\
& =1 & {[(\pi / 2)<|\omega|<\pi] .}
\end{array}
$$

If Eq. (3.6) is multiplied by $P_{k}^{1}(\cos \omega) \sin \omega$ and integrated from 0 to $\pi$, all terms in the series except the $k$

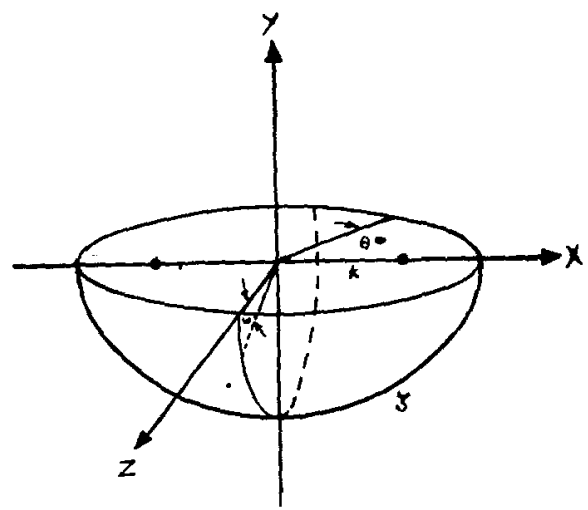

FIG. 4. Water impact of an ellipsoid of revolution.

term vanish because of the orthogonality of Legendre polynomials. One, therefore, obtains:

$$
\begin{aligned}
\alpha_{k}=\frac{2 k+1}{2 k(k+1)}\left[\int_{0}^{\pi / 2} P_{k}^{1}(\cos \omega) \sin \omega d \omega\right. \\
\\
\left.\quad-\int_{A / 2}^{\pi} P_{k}^{1}(\cos \omega) \sin \omega d \omega\right]
\end{aligned}
$$

Since $P_{k}{ }^{1}(\cos \omega)=\sin \omega\left[d P_{k}(\cos \omega) / d(\cos \omega)\right]$, this is rewritten, with due regard to symmetry properties:

$$
\begin{aligned}
\alpha_{2 k} & =\frac{4 k+1}{2 k(2 k+1)} \int_{0}^{\pi / 2} \sin ^{2} \omega P_{2 k}{ }^{\prime}(\cos \omega) d \omega . \\
\alpha_{2 k+1} & =0
\end{aligned}
$$

By use of Rodrigues's Formula ${ }^{11}$ the Legendre polynomial $P_{2 k}(z)$ can be written as:

$P_{2 k}(z)=\frac{1}{2^{k} k !} \sum_{r=0}^{k}\left(\begin{array}{c}k-r \\ r\end{array}\right) \frac{(2 k+2 r-1) ! !}{(2 r-1) ! !}(-)^{k-r} z^{2 r}$.

It is also known ${ }^{12}$ that

$$
\int_{0}^{\pi / 2} \sin ^{2} \omega \cos ^{2 r-1} \omega d \omega=\frac{(2 r-2) ! !}{(2 r+1) ! !}
$$

${ }^{11}$ Whittaker and Watson, Modern Analysis (Cambridge University Press, London, 1940), fourth edition.

$12 \mathrm{H}$. B. Dwight, Tables of Integrals and Other Mathematical Data (MacMillan Company, Ltd., London, 1934). 
Combining formulas (3.8a) and (3.8b), one obtains:

$$
\begin{aligned}
\alpha_{2 k}= & \frac{4 k+1}{2^{k} k(k !)(2 k+1)} \sum_{r=1}^{k}(-)^{k-r+1} \\
& \quad \times\left(\begin{array}{c}
k-r \\
k
\end{array}\right) \frac{r(2 r-2) ! !(2 k+2 r-1) ! !}{(2 r-1) ! !(2 r+1) ! !} .
\end{aligned}
$$

Thus, for instance :

$$
\begin{array}{ll}
\alpha_{2}=5 / 6=0.833 & \alpha_{8}=-0.041 \\
\alpha_{4}=-(3 / 40)=-0.075 & \alpha_{10}=0.0658 . \\
\alpha_{6}=0.147 & \alpha_{12}=-0.0094 \cdots
\end{array}
$$

The convergence of the series (3.6) is established by noting that the integral in $(3.7 \mathrm{~b})$ can be determined asymptotically as $k \rightarrow \infty$ as follows :

$$
\begin{gathered}
\lim _{k \rightarrow \infty} \int_{0}^{\pi / 2} P_{2 k^{\prime}}(\cos \omega) \sin ^{2} \omega d \omega \\
=\int_{0}^{\pi / 2} \sin ^{2} \omega \lim _{k \rightarrow \infty} P_{2 k}{ }^{\prime}(\cos \omega) d \omega \\
=\int_{0}^{\pi / 2} \sin ^{2} \omega(k / \pi)^{1} \sin 2 k \omega d \omega \\
=\frac{1}{4}(k / \pi)^{\frac{1}{2}} \int_{0}^{\pi}(1-\cos \theta) \sin k \theta d \theta \sim 1 /(k)^{1} .
\end{gathered}
$$

The general coefficient $\alpha_{k}$ therefore behaves as $k^{-t}$. Since ${ }^{13}$

$$
\begin{aligned}
\lim _{k \rightarrow \infty} P_{2 k}{ }^{1} & (\cos \theta) \\
& =-2(k / \pi \sin \theta)^{\frac{1}{2}} \sin \left[\left(2 k+\frac{1}{2}\right) \theta+3 \pi / 4\right]
\end{aligned}
$$

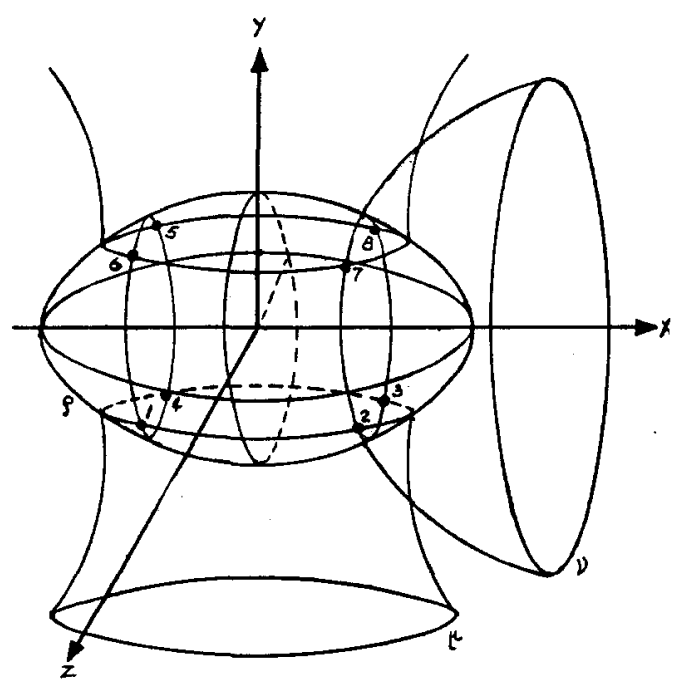

Fig. 5. Coordinate system for a general ellipsoid.

${ }^{13}$ E. W. Hobson, The Theory of Spherical and Ellipsoidal Harmonics (Cambridge University Press, London, 1931), Chapter 11. the series (3.6) behaves as $\sum\left(e^{i k \theta} / k\right)$ which is known to converge.

The potential of the horizontal flow past a sphere of radius $R$ is, therefore,

$$
\varphi_{1}=R \cos \theta \sum_{1}^{\infty}(R / r)^{n+1}\left(\alpha_{n} / n+1\right) P_{n}^{1}(\cos \omega) .
$$

The separation point is reached along the curve $\theta(\omega)$ where the following relation holds:

$\theta=\sec ^{-1}\left[-2 \cot \alpha \sec \omega \sum_{1}^{\infty}\left(\alpha_{n} / n+1\right) P_{n}^{1}(\cos \omega)\right]$.

If separation effects are neglected, one finds the following momentum integrals:

$$
\begin{aligned}
M_{x} & =(\pi / 2) \rho R^{3} \sum_{0}^{\infty}\left(\alpha_{n}\right)^{2} \\
M_{x y} & =M_{y x}=0 \\
M_{y} & =(\pi / 3) \rho R^{3} .
\end{aligned}
$$

\section{HALF AN ELLIPSOID OF REVOLUTION}

A slight generalization is obtained if the hemisphere considered above is replaced by an ellipsoid of revolution whose axis is parallel to the horizontal velocity component (Fig. 4). The potential problem is most simply discussed in terms of elliptic coordinates. If $x, y, z$, are cartesian coordinates as before, and the distance between the foci of the ellipsoid is $2 k$, the new coordinates $\mu, \zeta, \omega$ are defined by

$$
\begin{aligned}
& x=k \mu \zeta \\
& y=k\left[\left(1-\mu^{2}\right)\left(\zeta^{2}-1\right)\right]^{\frac{1}{3}} \sin \omega \\
& z=k\left[\left(1-\mu^{2}\right)\left(\zeta^{2}-1\right)\right]^{\frac{1}{3}} \cos \omega .
\end{aligned}
$$

The ellipsoid under study is defined by a value $\zeta_{0}$ of the parameter $\zeta$, and solutions are sought in the domain $\zeta>\zeta_{0}$ which corresponds to the space outside the ellipsoid.

The boundary conditions corresponding to $(1.8 \mathrm{a}, \mathrm{b})$ are here:

$$
\begin{aligned}
& \left.\frac{\partial \varphi_{1}}{\partial \zeta}\right)_{\zeta_{0}}=-k \mu \frac{|\cos \omega|}{\cos \omega} \\
& \left.\frac{\partial \varphi_{2}}{\partial \zeta}\right)_{\zeta_{0}}=-k \zeta_{0}\left(\frac{1-\mu^{2}}{\zeta_{0}{ }^{2}-1}\right)^{\frac{1}{2}} \sin \omega
\end{aligned}
$$

The potential $\varphi_{2}$ is known to be $\mathrm{e}^{11}$

$$
\begin{aligned}
\varphi_{2}= & \frac{k}{\frac{1}{2} \log \left(\zeta_{0}+1 / \zeta_{0}-1\right)-\left[\zeta_{0}^{2}-2 / \zeta_{0}\left(\zeta_{0}^{2}-1\right)\right]} \\
& \times\left\{\frac{1}{2} \log \frac{\zeta+1}{\zeta-1}-\frac{\zeta}{\zeta^{2}-1}\right\}\left[\left(1-\mu^{2}\right)\left(\zeta^{2}-1\right)\right]^{3} \cos \omega .
\end{aligned}
$$


The general potential convergent for $\zeta>\zeta_{0}$ is

$$
\varphi_{1}=\sum_{0}^{\infty} \sum_{1}^{n+1} Q_{n}{ }^{s}(\zeta) P_{n}{ }^{*}(\mu)\left[a_{n}{ }^{*} \sin s \omega+b_{n} \cdot \cos s \omega\right]
$$

so that boundary condition (4.2a) gives:

$$
\begin{aligned}
\sum_{0}^{\infty} \sum_{1}^{n+1} Q_{n}{ }^{8}\left(\zeta_{0}\right) P_{n}{ }^{8}(\mu) a_{n}{ }^{8} \sin s \omega & \\
\Rightarrow \sum_{0}^{\infty} \sum_{1}^{n+1} \alpha_{n}^{8} P_{n}^{8}(\mu) \sin s \omega & =-\mu(-\pi<\omega<0) \\
& =\mu(0<\omega<\pi)
\end{aligned}
$$

from which the coefficients $\alpha_{n}{ }^{\prime}$ are determined. Multiplication of (4.5a) by $\sin (r \omega)$ and integration between $-\pi$ and $\pi$ leads to:

$$
\begin{gathered}
\sum_{0}^{\infty} \alpha_{n}^{2 r-1} P_{n}^{2 r-1}(\mu)=\frac{4(-)^{r} \mu}{\pi} \frac{1}{2 r+1} \\
\alpha_{n}^{2 r}=0 .
\end{gathered}
$$

Multiplication of $(4.5 \mathrm{~b})$ by $P_{k}^{2 r+1}(\mu)$ and integration between -1 and 1 leads to:

$$
\begin{gathered}
\alpha_{2 k}^{2 r-1}=\frac{(-)^{r} 8(4 k+1)(2 k-2 r-1) !}{\pi(2 r+1)(2 k+2 r+1) !} \\
\times \int_{0}^{1} \mu P_{2 k}^{2 r+1}(\mu) d \mu \\
\alpha_{2 k+1}{ }^{2 r+1}=0 .
\end{gathered}
$$

After two integrations by parts, the integral in (4.5c) becomes:

$$
\begin{gathered}
\int_{0}^{1} \mu P_{2 k^{2 r+1}}(\mu) d \mu=\int_{0}^{1} \mu\left(1-\mu^{2}\right)^{\frac{1}{2}} \frac{d}{d \mu} P_{2 k^{2 r}}(\mu) d \mu \\
=\int_{0}^{1} \frac{2 \mu^{2}-1}{\left(1-\mu^{2}\right)^{\frac{1}{2}}} P_{2 k}{ }^{2 r}(\mu) d \mu \\
=\int_{0}^{1}\left(2 k^{2}-1\right) \frac{d}{d \mu} P_{2 k^{2 r-1}}(\mu) d \mu \\
=-4 \int_{0}^{1} \mu P_{2 k}^{2 r-1}(\mu) d \mu
\end{gathered}
$$

since

$$
P_{2 k}^{2 r+1}(0)=P_{2 k}^{2 r+1}(1)=0 .
$$

The recursion formula (4.6a) gives at once:

$$
\begin{aligned}
\int_{0}^{1} \mu P_{2 k}^{2 r+1}(\mu) d \mu & =(-4)^{r} \int_{0}^{1} \mu P_{2 k}^{1}(\mu) d \mu \\
& =(-4)^{r} \int_{0}^{\pi / 2} \sin ^{2} \theta P_{2 k}(\cos \theta) d \theta
\end{aligned}
$$

By using the representation (3.8a) for $P_{2 k}(z)$ and the relation:

$$
\int_{0}^{\pi / 2} \sin ^{2} \theta \cos ^{2} \theta d \theta=\frac{\pi}{2} \frac{(2 p-1) ! !}{(2 p+2) ! !}
$$

one finds the coefficient $\alpha_{2 k}{ }^{2 r+1}$

$$
\begin{aligned}
\alpha_{2 k}^{2 r+1}= & \frac{4^{r+1}}{2^{k-1} k !} \frac{4 k+1}{2 r+1} \frac{(2 k-2 r-1) !}{(2 k+2 r+1) !} \\
& \times \sum_{p=1}^{k}(-)^{k-p+1}\left(\begin{array}{c}
k-p \\
p
\end{array}\right) \frac{p(2 k+2 p-1) ! !}{(2 p+2) ! !}
\end{aligned}
$$

Thus for instance:

$$
\begin{aligned}
& \alpha_{2}{ }^{1}=1.250 \\
& \alpha_{4}{ }^{1}=0.281 \\
& \alpha_{6}{ }^{1}=0.127 \\
& \alpha_{8}{ }^{1}=0.0726 \\
& \alpha_{4}{ }^{3}=0.00161 \\
& \alpha_{6}{ }^{3}=0.00002 \\
& \alpha_{6}^{5}=0.00001 \text {. } \\
& \alpha_{10}{ }^{1}=0.0471 \\
& \alpha_{12}{ }^{1}=0.0328
\end{aligned}
$$

The uniform convergence of the doubly infinite series (4.5a) is established by the Weierstrass $M$ test if $\sum \sum \alpha_{k}{ }^{r}$ converges: The integral in (4.5c) converges:

$$
\lim _{k \rightarrow \infty}\left|\int_{0}^{1} \mu P_{2 k}{ }^{\mathrm{I}} d \mu\right|<\frac{A}{(k)^{\frac{1}{2}}}
$$

as can be shown by an argument similar to that of (3.10). For any value of $r$, therefore, the series

$$
\sum_{k=0}^{\infty} \alpha_{k}^{r}=S^{\prime}(r)
$$

converges absolutely. For any given value of $k$,

$$
\lim _{r \rightarrow \infty} \sum_{0}^{k} \alpha_{k}^{r}<A(k) \sum \frac{4^{r}}{(2 r+1)(4 r) !}
$$

so that the sum of rows as well as the sum of columns converges absolutely. Then, one has

$$
\left|\sum \sum \alpha_{k}{ }^{r}\right|<\sum \sum(1 /(k+r) !)
$$

which establishes the convergence of the series. The potential $\varphi_{1}$ for the horizontal flow past the halfellipsoid of revolution is, therefore:

$$
\begin{aligned}
\varphi_{1}=\sum_{n=1}^{\infty} \sum_{s=0}^{n-1} \frac{Q_{2 n^{2 \delta+1}}(\zeta)}{Q_{2 n}{ }^{2 \delta+1}\left(\zeta_{0}\right)} \\
\quad \times \alpha_{2 n^{28+1}} P_{2 n^{28+1}}(\mu) \sin (2 s+1) \omega .
\end{aligned}
$$

The momentum integrals are:

$$
\begin{aligned}
M_{z}=\frac{\pi}{4} k^{3}\left(\zeta_{0}^{2}-1\right) \sum_{n=1}^{\infty} \sum_{s=1}^{n-1} \frac{Q_{2 n^{2 o+1}}\left(\zeta_{0}\right)}{Q_{2 n}{ }^{2 s+1}\left(\zeta_{0}\right)} \\
\\
\times \frac{(2 n+2 s+1) !\left(\alpha_{2 n}{ }^{2 s+1}\right)^{2}}{(2 n-2 s-1) ! 4 n+1}
\end{aligned}
$$




$$
\begin{aligned}
M_{x y} & =M_{y x}=0 \\
M_{\nu} & =\frac{\pi k^{3}}{2} \zeta_{0}\left(\zeta_{0}^{2}-1\right) \\
& \times \frac{\log \left(\zeta_{0}+1 / \zeta_{0}-1\right)-\left(2 \zeta_{0} / \zeta_{0}^{2}-1\right)}{\log \left(\zeta_{0}+1 / \zeta_{0}-1\right)-\left[2\left(\zeta_{0}^{2}-2\right) / \zeta_{0}\left(\zeta_{0}^{2}-1\right)\right]} .
\end{aligned}
$$

Note that the entire argument was carried through for a prolate ellipsoid $(\zeta>1)$. If the ellipsoid is oblate

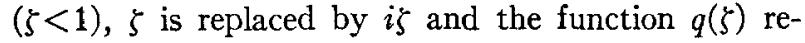
places $Q(\zeta)$, where

$$
g_{n}{ }^{s}(\zeta)=Q_{n}{ }^{n}(i \zeta)
$$

everything else remaining unchanged.

\section{HALF A GENERAL ELLIPSOID}

A further generalization is obtained by considering an ellipsoid with focal length $2 k$ parallel to the horizontal velocity component, and focal length $2 h$ horizontal and normal to it. The problem is now formulated in terms of ellipsoidal coordinates defined by $\mathrm{E}$. W. Hobson: $:^{13}$

$$
\begin{aligned}
& x=\rho \mu \nu / h k \\
& y=\frac{\left[\left(\rho^{2}-h^{2}\right)\left(\mu^{2}-h^{2}\right)\left(h^{2}-\nu^{2}\right)\right]^{\frac{1}{3}}}{h\left(k^{2}-h^{2}\right)^{\frac{1}{2}}} \\
& z=\frac{\left[\left(\rho^{2}-k^{2}\right)\left(k^{2}-\mu^{2}\right)\left(k^{2}-\nu^{2}\right)\right]^{\frac{1}{2}}}{k\left(k^{2}-h^{2}\right)^{\frac{1}{2}}} .
\end{aligned}
$$

The surfaces $\rho=$ const. represent confocal ellipsoids; $\rho_{0}$ in particular represents the ellipsoid under investigation; $\mu=$ const. are hyperboloids of one sheet and $\nu=$ const. are hyperboloids of two sheets. The coordinate system is shown on Fig. 5. Any point is defined uniquely if the signs of the radicals in (5.1) are defined. Boundary conditions $(1.8 \mathrm{a}, \mathrm{b})$ become in this system :

$$
\begin{aligned}
& \left.\frac{\partial \varphi_{1}}{\partial \rho}\right)_{\rho_{0}}=-\frac{\mu \nu}{h k} \frac{|y|}{y} \\
& \left.\frac{\partial \varphi_{2}}{\partial \rho}\right)_{\rho_{0}}=-\frac{\rho_{0}}{h}\left\{\frac{\left[\left(\mu^{2}-h^{2}\right)\left(h^{2}-\nu^{2}\right)\right]}{\left[\left(\rho_{0}^{2}-h^{2}\right)\left(k^{2}-h^{2}\right)\right]}\right\}^{\frac{1}{2}} .
\end{aligned}
$$

The potential $\varphi_{2}$ is given explicitly in the standard literature ${ }^{11}$ by the formula:

$\varphi_{2}=\frac{\rho_{0}}{2 h}\left[\frac{\left(\rho_{0}^{2}-h^{2}\right)\left(\rho_{0}^{2}-k^{2}\right)}{k^{2}-h^{2}}\right]^{\frac{1}{2}}$

$$
\begin{gathered}
\times \frac{1}{1-\rho_{0}\left[\left(\rho_{0}^{2}-h^{2}\right)\left(\rho_{0}^{2}-k^{2}\right)\right]^{\frac{1}{2}} \int_{\rho_{0}}^{\infty} \frac{d \rho}{\left(\rho^{2}-h^{2}\right)^{\frac{1}{2}}\left(\rho^{2}-k^{2}\right)^{\frac{1}{1}}}} \\
\times\left[\left(\rho^{2}-h^{2}\right)\left(\mu^{2}-h^{2}\right)\left(h^{2}-\nu^{2}\right)\right]^{\frac{1}{2}} \\
\times \int_{\rho}^{\infty} \frac{d \rho}{\left(\rho^{2}-h^{2}\right)^{\frac{1}{2}}\left(\rho^{2}-k^{2}\right)^{\frac{1}{2}}}
\end{gathered}
$$

The general potential convergent for $\rho>\rho_{0}$ is

$$
\varphi_{1}=\sum_{0}^{\infty} \sum_{1}^{2 n+1} a_{n}{ }^{s} F_{n}^{s}(\rho) E_{n}^{s}(\mu) E_{n}{ }^{s}(\nu),
$$

where $F_{n}{ }^{s}, E_{n}{ }^{s}$ are general Lamé functions, convergent as $\rho \rightarrow \infty$ and $\mu, \nu \rightarrow 0$, and satisfying Lamé's differential equation:

$$
\begin{gathered}
\left(\lambda^{2}-h^{2}\right)\left(\lambda^{2}-k^{2}\right)\left(d^{2} E_{n} / d \lambda^{2}\right) \\
+\lambda\left(2 \lambda^{2}-h^{2}-k^{2}\right)\left(d E_{n} / d \lambda\right) \\
+\left[\left(h^{2}+k^{2}\right) p-n(n+1) \lambda^{2}\right] E_{n}=0 \\
\lambda=\rho, \mu, \nu
\end{gathered}
$$

the parameter $n$ is an integer, while $p(h, k, n)$ is chosen so that $E_{n}$ is a polynomial. The theory of Lamé functions is discussed in detail in. ${ }^{13}$

Boundary condition (5.2a) gives here:

$$
\begin{aligned}
h k \sum_{0}^{\infty} \sum_{1}^{2 n+1} F_{n}{ }^{s}\left(\rho_{0}\right) a_{n}{ }^{s} E_{n}{ }^{s}(\mu) E_{n}{ }^{s}(\nu) & \\
=\sum_{0}^{\infty} \sum_{1}^{2 n+1} \alpha_{n}^{s} E_{n} s(\mu) E_{n}{ }^{s}(\nu) & =\mu \nu\left[\left(\mu^{2}-h^{2}\right)^{\frac{1}{2}}>0\right] \\
& =-\mu \nu\left[\left(\mu^{2}-h^{2}\right)^{\frac{1}{2}}<0\right] .
\end{aligned}
$$

From the form of the function to be expanded, it is clear that only odd Lamé functions $K_{2 n+1}{ }^{8}$ of the form

$$
K_{2 n+1}{ }^{8}(\lambda)=\beta_{1}^{s} \lambda+\beta_{3}^{8} \lambda^{3}+\cdots \beta_{2 n+1}{ }^{8} \lambda^{2 n+1}
$$

will appear in $\varphi_{1}$. The coefficients $\beta_{n}{ }^{s}$ are defined by recurrence formulas and depend on $(h, k, p)$. They have been tabulated by Guerritore. ${ }^{14}$ It is then known ${ }^{15}$ that the coefficients $\alpha_{n}{ }^{s}$ are given by:

$$
\alpha_{2 n+1} s=\frac{\int_{0}^{h} \int_{h}^{k}\left(\mu^{2}-\nu^{2}\right) \mu \nu K_{2 n+1^{s}}(\mu) K_{2 n+1}{ }^{s}(\nu) \frac{d \mu}{\left[\left(h^{2}-\mu^{2}\right)\left(\mu^{2}-k^{2}\right)\right]^{\frac{1}{2}}} \frac{d \nu}{\left[\left(h^{2}-\nu^{2}\right)\left(k^{2}-\nu^{2}\right)\right]^{\frac{1}{2}}} .}{\int_{0}^{h} \int_{h}^{k}\left(\mu^{2}-\nu^{2}\right)\left[K_{2 n+1}{ }^{s}(\mu) K_{2 n+1^{s}}(\nu)\right]^{2} \frac{d \mu}{\left[\left(h^{2}-\mu^{2}\right)\left(\mu^{2}-k^{2}\right)\right]^{\frac{1}{2}}} \frac{d \nu}{\left[\left(h^{2}-\nu^{2}\right)\left(k^{2}-\nu^{2}\right)\right]^{\frac{1}{2}}}} .
$$

${ }^{14}$ M. Guerritore, Table of Lamé functions, Giornale de Matematica (2) XVI, 164-172 (1909).

15 O. Volk, "Uber die entwicklung von functionen zweier complexen veranderlichen nach lameschen functionen," Math. Zeitsch 23, 224 (1925). 
If the expansion (5.6) for $K_{2 n+1}{ }^{8}(\lambda)$ is substituted into the integrals (5.7), it is found that one must investigate integrals of the form:

$$
\begin{aligned}
& A_{2 r}(\lambda)=\int_{0}^{h} \frac{\lambda^{2 r} d \lambda}{\left[\left(h^{2}-\lambda^{2}\right)\left(k^{2}-\lambda^{2}\right)\right]^{\frac{1}{2}}} \\
& B_{2 r}(\lambda)=\int_{h}^{k} \frac{\lambda^{2 r} d \lambda}{\left[\left(\lambda^{2}-h^{2}\right)\left(k^{2}-\lambda^{2}\right)\right]^{\frac{1}{2}}} .
\end{aligned}
$$

It is easily verified that:

$$
\begin{aligned}
& A_{0}=(1 / k) F(h / k) \quad B_{0}=(1 / k) F\left[\frac{\left(k^{2}-h^{2}\right)^{\frac{3}{3}}}{k}\right](5.9 \mathrm{a}, \mathrm{b}) \\
& A_{2}=k(F(h / k)-E(h / k)) B_{2}=k E\left[\frac{\left(k^{2}-h^{2}\right)^{\frac{1}{3}}}{k}\right] . \quad(5.9 \mathrm{c}, \mathrm{d})
\end{aligned}
$$

Repeated integrations by parts then lead to the recurrence formulas:

$$
\begin{aligned}
(2 n-1) A_{2 n}=(2 n-4)\left(h^{2}+k^{2}\right) A_{2 n-2} & -(2 n-3) h^{2} k^{2} A_{2 n+4}
\end{aligned}
$$

and an identical result for $B_{2 n}$. The integrals $A_{2 n}, B_{2 n}$ are, therefore, expressed as functions of the parameters $h, k$ in terms of elliptic integrals.

The numerator and denominator of (5.7) are now simply expressed in terms of the known coefficients $\beta_{n}{ }^{8}$ and the integrals $A_{2 n}, B_{2 n}$.

$$
\begin{gathered}
\left.N_{2 n+1^{8}=\left(\beta_{1}^{8}\right)^{2}(} B_{4} A_{2}-B_{2} A_{4}\right) \\
+\beta_{1}^{8} \beta_{3}^{8}\left(B_{6} A_{2}-A_{6} B_{2}\right) \\
\quad+\left(\beta_{3}^{8}\right)^{2}\left(B_{6} A_{4}-B_{4} A_{6}\right) \cdots \\
D_{2_{n+1}}=\left(\beta_{1}^{8}\right)^{4}\left(B_{4} A_{2}-B_{2} A_{4}\right) \\
+2 \beta_{3}^{8}\left(\beta_{1}^{8}\right)^{3}\left(B_{6} A_{2}-A_{6} B_{2}\right) \cdots \\
\alpha_{2 n+1^{8}}=N_{2 n+1^{8}} / D_{2 n+1^{8}} .
\end{gathered}
$$

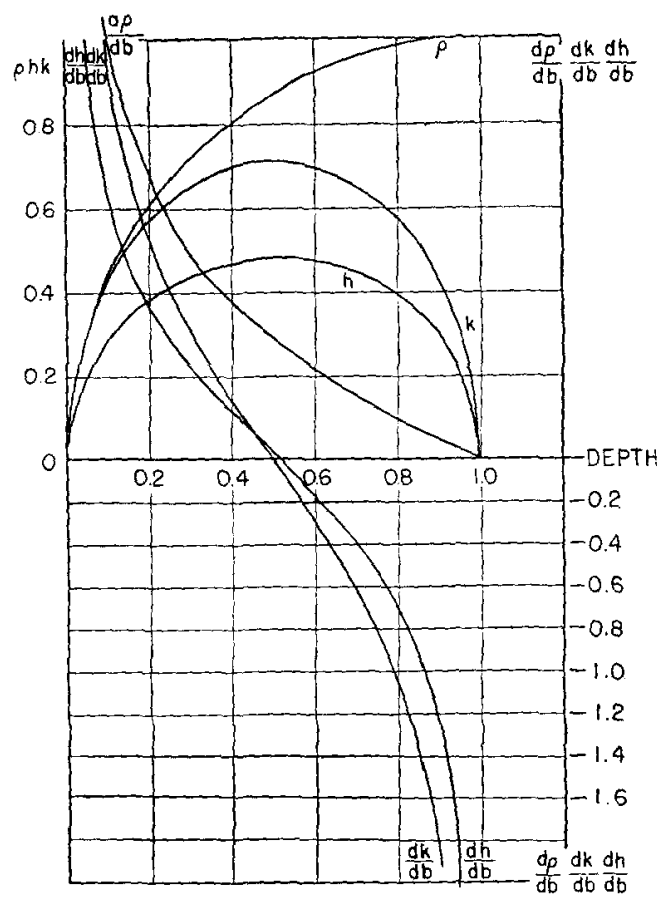

FIG. 6. The approximating functions for a partially submerged sphere and their derivatives.

The potential function $\varphi_{1}$ is then given by:

$$
\begin{aligned}
\varphi_{1}=\frac{1}{h k} \sum_{0}^{\infty} \sum_{1}^{n+1 / 2} \frac{F_{2 n+1}{ }^{s}(\rho)}{F_{2 n+1}{ }^{s}\left(\rho_{0}\right)} \\
\quad \quad \times \alpha_{2 n+1}{ }^{s} K_{2 n+1}{ }^{s}(\mu) K_{2 n+1}{ }^{8}(\nu) .
\end{aligned}
$$

The momentum integrals are:

$$
\begin{aligned}
& M_{x}=\frac{\left[\left(\rho_{0}{ }^{2}-h^{2}\right)\left(\rho_{0}{ }^{2}-k^{2}\right)\right]^{\frac{1}{2}}}{h^{2} k^{2}} \sum_{0}^{\infty} \sum_{1}^{n+1 / 2} \frac{F_{2 n+1^{s}}\left(\rho_{0}\right)}{F_{2 n+1}{ }^{s}\left(\rho_{0}\right)}\left(\alpha_{2 n+1^{s}}\right)^{2} D_{2 n+1}{ }^{s} \\
& M_{x y}=M_{y x}=0 \\
& M_{y}=\left(\pi \rho_{0} / 12\right)\left[\left(\rho_{0}^{2}-h^{2}\right)\left(\rho_{0}^{2}-k^{2}\right)\right]^{\frac{1}{2}-} \frac{\rho_{0}\left[\left(\rho_{0}{ }^{2}-h^{2}\right)\left(\rho_{0}{ }^{2}-k^{2}\right)\right]^{\frac{1}{2}} \int_{\rho_{0}}^{\infty} \frac{d \rho}{\rho_{0}\left(\rho^{2}-h^{2}\right)^{\frac{3}{3}}\left(\rho^{2}-k^{2}\right)^{\frac{1}{3}}}}{1-\rho_{0}\left[\left(\rho_{0}^{2}-h^{2}\right)\left(\rho_{0}{ }^{2}-k^{2}\right)\right]^{\frac{1}{2}} \int_{\rho_{0}}^{\infty} \frac{d \rho}{\rho_{0}\left(\rho^{2}-h^{2}\right)^{\frac{3}{2}}\left(\rho^{2}-k^{2}\right)^{\frac{1}{2}}}} .
\end{aligned}
$$

The first three terms of this expansion were computed numerically and used in Section 6.

\section{WATER IMPACT OF A SPHERE AT AN ENTRY ANGLE OF $45^{\circ}$}

The results of the previous pages are now applied to a specific problem. The drag on a sphere of radius $R$ which strikes the water surface at $45^{\circ}$ is computed as a function of time. The submerged portion of the sphere is approximated by a half-ellipsoid in such a manner that the depth of penetration $d$ of the sphere equals the minor axis, the diameter of the circle of intersection between the sphere and the free surface equals the major axis and the submerged volume of the sphere, $V$, equal the volume of the semi-ellipsoid. If the dimensionless length $\xi$ is introduced, one has:

$$
\begin{aligned}
\xi & =t V \sin \alpha / R \\
\rho_{0}(t) / R & =[\xi(2-\xi)]^{\frac{1}{2}} \\
k(t) / R & =[2 \xi(1-\xi)]^{\frac{1}{2}} \\
h(t) / R & =\frac{1}{2}\left[\frac{\xi(1-\xi)(7-3 \xi)}{2-\xi}\right]^{\frac{1}{2}} \\
d \alpha_{i} / d t & =(V \sin \alpha / R)\left(d \alpha_{i} / d \xi\right) .
\end{aligned}
$$




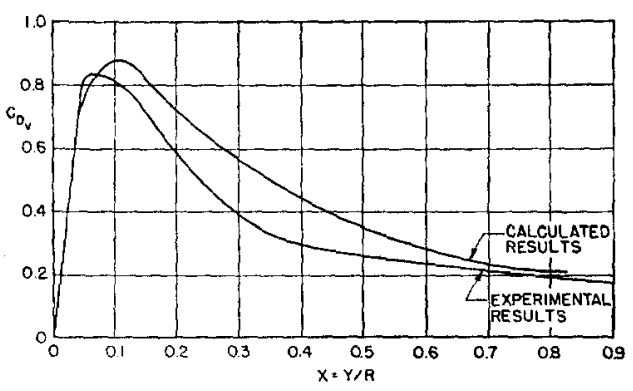

FIG. 7. Vertical component of drag of a sphere of unit radius in water impact $C_{D}=F_{Y} / \pi R^{2} \rho\left(V^{2} / 2\right)$.

The force functions are given by formulas $(1.10 \mathrm{a}, \mathrm{b})$ and the derivatives are given explicitly as:

$$
\begin{aligned}
& d \rho_{0} / d t=V \sin \alpha \frac{1-\xi}{2[\xi(2-\xi)]^{\frac{1}{2}}} \\
& d k / d t=V \sin \alpha \frac{1-2 \xi}{[2 \xi(1-\xi)]^{\frac{1}{2}}} \\
& d h / d t=V \sin \alpha \frac{7-20 \xi+14 \xi^{2}-3 \xi^{3}}{2\left[\xi(1-\xi)(7-3 \xi)(2-\xi)^{3}\right]^{\frac{1}{2}}}
\end{aligned}
$$

while the derivatives $\partial M / \partial \alpha_{i}$ are obtained by differentiating (5.13) with respect to $\rho_{0}, h, k$. This formulation is valid only while $\xi<1$, or up to the time when the sphere is half-submerged. After that instant, flow past an ellipsoid does not give a good approximation to the streamline pattern, and a cavity is beginning to develop.

The results of the calculation for the sphere are shown on Figs. 6, 7, 8. It is found that the value of the

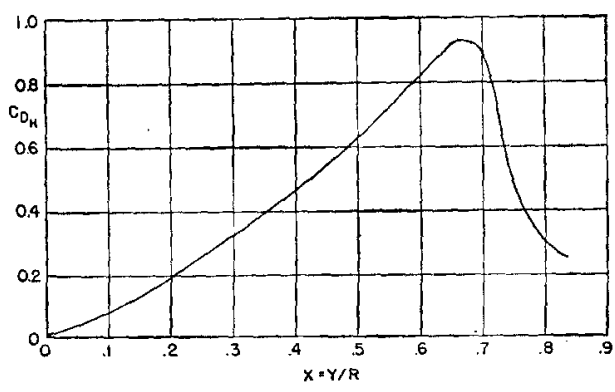

Fig. 8. Horizontal component of drag of a sphere in water impact.

vertical drag component agrees fairly well with the value obtained in unpublished experiments carried out at the Morris Dam Hydrodynamics Station; it also gives the same type of result as that obtained by Schiffman and Spencer. The horizontal component of drag reaches its greatest value at a later point.

\section{CONCLUSION}

The impact of a body of arbitrary shape at an arbitrary angle was investigated by approximate methods. The two main approximations consisted in linearizing the boundary condition at the free surface by neglecting the splash and in replacing the submerged portion of the body at any time by an ellipsoid of identical depth of penetration, length, and submerged volume. As an illustration of the method, the impact of a sphere at a $45^{\circ}$ angle was studied in some detail. The vertical force component turned out to be in good agreement with experimental results. The behavior of the horizontal component, which has not been measured, was also computed and shown. 